\title{
${ }^{18}$ F-DCFBC PET/CT for PSMA-Based Detection and Characterization of Primary Prostate Cancer
}

\author{
Steven P. Rowe*1, Kenneth L. Gage*1, Sheila F. Faraj ${ }^{2}$, Katarzyna J. Macura ${ }^{1,3,4}$, Toby C. Cornish ${ }^{2}$, Nilda Gonzalez- \\ Roibon², Gunes Guner ${ }^{2}$, Enrico Munari², Alan W. Partin ${ }^{3}$, Christian P. Pavlovich ${ }^{3}$, Misop Han ${ }^{3}$, H. Ballentine Carter ${ }^{3}$, \\ Trinity J. Bivalacqua ${ }^{3}$, Amanda Blackford ${ }^{1}$, Daniel Holt ${ }^{1}$, Robert F. Dannals ${ }^{1}$, George J. Netto ${ }^{2-4}$, Martin A. Lodge ${ }^{1}$, \\ Ronnie C. Mease ${ }^{1}$, Martin G. Pomper ${ }^{1}$, and Steve Y. Cho ${ }^{1,4}$ \\ ${ }^{I}$ The Russell H. Morgan Department of Radiology and Radiological Science, Johns Hopkins Medical Institutions, Baltimore, \\ Maryland; ${ }^{2}$ Department of Pathology, Johns Hopkins Medical Institutions, Baltimore, Maryland; ${ }^{3}$ The James Buchanan Brady \\ Urological Institute and Department of Urology, Johns Hopkins Medical Institutions, Baltimore, Maryland; and ${ }^{4}$ Sidney Kimmel \\ Comprehensive Cancer Center, Johns Hopkins Medical Institutions, Baltimore, Maryland
}

We previously demonstrated the ability to detect metastatic prostate cancer using $N$-[N-[(S)-1,3-dicarboxypropyl]carbamoyl]4-18 F-fluorobenzyl-L-cysteine ( ${ }^{18} \mathrm{~F}$-DCFBC), a low-molecular-weight radiotracer that targets the prostate-specific membrane antigen (PSMA). PSMA has been shown to be associated with higher Gleason grade and more aggressive disease. An imaging biomarker able to detect clinically significant high-grade primary prostate cancer reliably would address an unmet clinical need by allowing for riskadapted patient management. Methods: We enrolled 13 patients with primary prostate cancer who were imaged with ${ }^{18} \mathrm{~F}$-DCFBC PET before scheduled prostatectomy, with 12 of these patients also undergoing pelvic prostate MR imaging. Prostate ${ }^{18} \mathrm{~F}-\mathrm{DCFBC}$ PET was correlated with MR imaging and histologic and immunohistochemical analysis on a prostate-segment (12 regions) and dominantlesion basis. There were no incidental extraprostatic findings on PET suggestive of metastatic disease. Results: MR imaging was more sensitive than ${ }^{18} \mathrm{~F}-\mathrm{DCFBC}$ PET for detection of primary prostate cancer on a per-segment (sensitivities of up to 0.17 and 0.39 for PET and MR imaging, respectively) and per-dominant-lesion analysis (sensitivities of 0.46 and 0.92 for PET and MR imaging, respectively). However, ${ }^{18} \mathrm{~F}-\mathrm{DCFBC}$ PET was more specific than MR imaging by per-segment analysis (specificities of 0.96 and 0.89 for PET and MR imaging for corresponding sensitivity, respectively) and specific for detection of high-grade lesions (Gleason 8 and 9) greater than $1.0 \mathrm{~mL}$ in size (4/4 of these patients positive by PET). ${ }^{18} \mathrm{~F}$-DCFBC uptake in tumors was positively correlated with Gleason score ( $\rho=0.64$; PSMA expression, $\rho=0.47$; and prostate-specific antigen, $\rho=0.52$ ). There was significantly lower ${ }^{18} \mathrm{~F}$-DCFBC uptake in benign prostatic hypertrophy than primary tumors (median maximum standardized uptake value, 2.2 vs. $3.5 ; P=0.004$ ). Conclusion: Although the sensitivity of ${ }^{18} \mathrm{~F}-\mathrm{DCFBC}$ for primary prostate cancer was less than MR imaging, ${ }^{18} \mathrm{~F}$-DCFBC PET was able to detect the more clinically significant high-grade and larger-volume tumors (Gleason score 8 and 9) with higher specificity than MR imaging. In particular, there was relatively low ${ }^{18} \mathrm{~F}-\mathrm{DCFBC}$ PET uptake in benign prostatic hypertrophy lesions, compared with cancer in the prostate, which may allow for more specific detection of primary prostate cancer by ${ }^{18} \mathrm{~F}-\mathrm{DCFBC}$

Received Jan. 13, 2015; revision accepted May 7, 2015.

For correspondence or reprints contact: Steve Y. Cho, 1111 Highland Ave., WIMR 7139, Madison, WI 53705.

E-mail: scho@uwhealth.org

${ }^{*}$ Contributed equally to this work.

Published online Jun. 11, 2015.

COPYRIGHT (c) 2015 by the Society of Nuclear Medicine and Molecular Imaging, Inc.
PET. This study demonstrates the utility of PSMA-based PET, which may be used in conjunction with MR imaging to identify clinically significant prostate cancer.

Key Words: primary prostate cancer; prostate specific membrane antigen (PSMA); PET/CT; MRI; prostatectomy

J Nucl Med 2015; 56:1003-1010

DOI: 10.2967/jnumed.115.154336

$\mathbf{I}$ the United States, prostate cancer remains the most commonly diagnosed cancer and second leading cause of cancer-related death in men (1). Accurate detection and appropriate management of patients with suspected primary prostate cancer is both challenging and controversial from clinical and imaging perspectives (2). Differentiation of indolent, low-risk from aggressive, high-risk, and potentially lethal disease at initial diagnosis represents an unmet clinical need. Development of new methods allowing appropriate risk stratification to conservative management, such as active surveillance programs, or definitive therapy, such as prostatectomy, radiotherapy, or up-front androgen-deprivation, are necessary (3). Incorporation of imaging to current primary prostate cancer classifications for risk stratification can help achieve that unmet clinical need.

Multiparametric prostate MR imaging, by combining anatomic and functional information, is emerging as an accurate tool for detection of primary prostate cancer compared with the traditional, 12-core random, transrectal ultrasound-guided technique, particularly in its ability to detect such aggressive features as capsular and seminal vesicle invasion $(4,5)$. However, MR imaging suffers from false-positives in nodules of benign prostatic hyperplasia $(\mathrm{BPH})$, which can have an imaging appearance that overlaps with prostate adenocarcinoma (6). Furthermore, small foci of disease, low-grade disease, and disease within the central gland may all be occult with MR imaging (5).

PET imaging has become an important functional imaging tool for diagnosis and staging in oncology, and several PET radiotracers have been developed and are being evaluated for detection and localization of primary prostate cancer $(7,8) .{ }^{18} \mathrm{~F}$-FDG is generally limited for the detection of primary prostate cancer. Other PET radiotracers evaluated for detection of primary prostate cancer include various agents targeting fatty acid metabolism $\left({ }^{11} \mathrm{C}\right.$-choline, ${ }^{18} \mathrm{~F}$-choline, ${ }^{11} \mathrm{C}$-acetate) (9-12) and amino acid transport (anti-1amino-3- ${ }^{18}$ F-fluorocyclobutane-1-carboxylic acid, or ${ }^{18} \mathrm{~F}-\mathrm{FACBC}$ ) 
$(13,14)$. Those radiotracers are able to demonstrate increased PET uptake at sites of primary prostate cancer, compared with normal prostate tissue, but generally have limited specificity for differentiating malignant from nonmalignant processes, such as BPH and prostatitis, and have low sensitivity for small tumors. Additional new PET radiotracers are being developed, including small molecules that target the prostate-specific membrane antigen (PSMA), gastrin-releasing peptide receptor, and glutamine, all of which show promise for imaging prostate cancer but have not been systematically evaluated for detection of primary disease (15-18).

PSMA is a rational and promising target for imaging prostate cancer given its high expression in this disease. Expression in primary and metastatic lesions is associated with tumor grade and clinical outcome (19-21). We have previously demonstrated the ability of $N-[N-[(S)-1,3$-dicarboxypropyl $]$ carbamoyl $]-4-{ }^{18}$ F-fluorobenzylL-cysteine ( ${ }^{18}$ F-DCFBC; Fig. 1) to bind to prostate tumors with high PSMA expression in preclinical studies (22) and in a first-in-human study, to localize prostate metastases to both bone and soft tissue accurately (23). In this prospective study, we further evaluated ${ }^{18} \mathrm{~F}$ DCFBC PET for detection and characterization of primary prostate cancer in men undergoing definitive surgery with correlation to pelvic MR imaging and pathology postprostatectomy.

\section{MATERIALS AND METHODS}

\section{Patient Population and Selection}

All studies were performed in accordance with the Johns Hopkins University Institutional Review Board under a Food and Drug Administration exploratory investigational new drug application (eIND 108943). Written, informed consent was obtained from all patients. Pertinent inclusion criteria for this study included newly diagnosed patients with biopsy-proven prostate cancer with a Gleason score of 6 or greater, considered as candidates for and medically fit to undergo prostatectomy, and at least $10 \mathrm{~d}$ after the most recent prostate biopsy at the time of PET imaging. Pertinent exclusion criteria included prior history of any other malignancy within the last $2 \mathrm{y}$ other than skin basal cell or nonmetastatic cutaneous superficial squamous cell carcinoma and superficial bladder cancer, history of prior radiation or systemic therapy for prostate cancer, renal dysfunction with serum creatinine greater than $1.5 \mathrm{mg} / \mathrm{dL}$ or creatinine clearance less than $50 \mathrm{~mL} / \mathrm{min} /$ $1.73 \mathrm{~m}^{2}$, and prostatectomy scheduled before a follow-up call that occurred within 12-72 $\mathrm{h}$ after imaging.

Fifteen patients were prospectively enrolled between September 2012 and April 2014. Thirteen patients were imaged with ${ }^{18}$ F-DCFBC $\mathrm{PET} / \mathrm{CT}$, with 12 of these patients also scanned with pelvic prostate

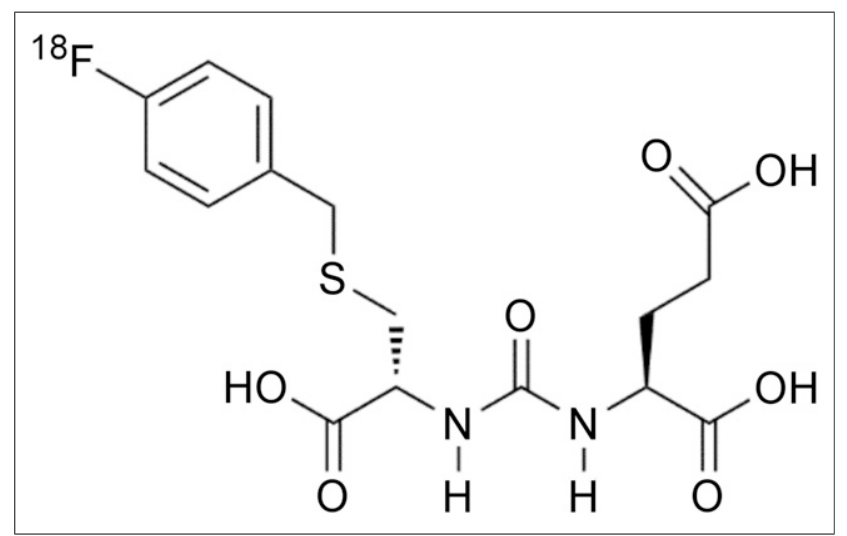

FIGURE 1. ${ }^{18} \mathrm{~F}-\mathrm{DCFBC}$ chemical structure.
MR imaging (1 patient was unable to undergo MR imaging because of a previously undisclosed remote history of foreign metal object). Two patients were enrolled but dropped out before any study-related ${ }^{18} \mathrm{~F}$ DCFBC administration or imaging.

\section{Radiochemistry}

2-[3-(1-carboxy-2-mercapto-ethyl)-ureido]-pentanedioic acid was prepared as previously described (24). Nonradiolabeled ${ }^{18}$ F-DCFBC precursors and standard were prepared according to a modification of a literature procedure to conform to current good manufacturing practice (25). ${ }^{18} \mathrm{~F}$-DCFBC was prepared as previously published $(23,26)$. The specific activity range of administered ${ }^{18} \mathrm{~F}$-DCFBC was $62.9-$ $2,084 \mathrm{GBq} / \mu$ mole $(1,699-56,318 \mathrm{mCi} / \mu$ mole $)$, with a median of $335 \mathrm{GBq} / \mu$ mole $(9,059 \mathrm{mCi} / \mu$ mole $)$. The variation in specific activity at time of injection may have been due in part to 1 or more of the following: starting amounts of ${ }^{18} \mathrm{~F}$-fluoride; carrier fluoride in target water, transfer tubings, and valves; minute and variable ultraviolet absorbing components from different batches of saline in the radiotracer product matrix used over the course of the study; and the interval between the end of synthesis and the time of injection (i.e., subject preparation and scanner availability).

\section{PET/CT Protocol}

Patients were instructed to be nil per os (except for water and some medications) for at least $6 \mathrm{~h}$ before the administration of ${ }^{18} \mathrm{~F}$-DCFBC. Patients were specifically asked not to take multivitamins and folic acid supplements on the day of the examination because folate is a substrate for PSMA and high folate levels can potentially reduce ${ }^{18} \mathrm{~F}-\mathrm{DCFBC}$ binding $(15,27)$. Serum folate was obtained before ${ }^{18} \mathrm{~F}-\mathrm{DCFBC}$ administration. A coudé catheter was placed in the urinary bladder as tolerated, which allowed for a consistent reference point between both PET and MR imaging in these patients, permitting improved PET and MR imaging coregistration.

PET/CT was performed on a Discovery DRX PET/CT scanner (GE Healthcare) operating in 2-dimensional (2D) and 3-dimensional (3D) emission acquisition mode with $\mathrm{CT}$ for attenuation correction. Scans were obtained with patients supine. A bolus of $370 \pm 37 \mathrm{MBq}(10 \pm 1$ $\mathrm{mCi})$ of ${ }^{18} \mathrm{~F}$-DCFBC was injected by slow intravenous push. Two hours after injection, a CT of the pelvis was obtained $(120 \mathrm{kVp}, 80$ $\mathrm{mA}$ maximum [auto-adjusting]), followed by a 30 -min dynamic listmode 2D PET emission acquisition, then an additional 5-min pelvic 3D PET, both with the prostate in the center of the field of view. Twodimensional PET acquisition mode was used for this pelvic PET imaging to minimize potential scatter caused by a PET quantitation artifact from the high urinary radioactivity in the bladder. Six 5-min frames were reconstructed with a high-resolution filter and summed to produce the 2D pelvic PET images. Pelvic PET images were acquired with the patient on a flat table with arms raised to allow for optimal coregistration with the corresponding MR images. After completion of the dynamic pelvic PET/CT scans at approximately $2.5 \mathrm{~h}$ after injection, a whole-body (WB) PET/CT was acquired from the vertex of the skull through the mid thigh in 3D mode for $4 \mathrm{~min}$ and $15 \mathrm{~s}$ at each bed position. All PET images were reconstructed using a standard clinical ordered-subset expectation maximization algorithm.

\section{MR Imaging Acquisition}

All pelvic MR imaging studies were obtained on the same day as the PET acquisitions except for 1 patient imaged $9 \mathrm{~d}$ later. MR imaging studies were performed on a 3-T WB TRIO scanner with a TIM body matrix coil (Siemens Medical Solutions). The sequences acquired included thin-section high-resolution axial, coronal, and sagittal T2-weighted fast spin-echo, diffusion-weighted imaging with corresponding apparent diffusion coefficient (ADC) maps from a 2D multislice single-shot diffusion-weighted echo planar sequence and a single-slab 3D isometric T2-weighted turbo spin-echo sequence (SPACE or sampling perfection with application-optimized contrast 
using different flip-angle evolution). The 3D T2-weighted SPACE sequence was acquired to allow high-fidelity coregistration with the PET and CT images given that its isometric voxels could be reconstructed in any needed plane. Axial T1 noncontrast images were obtained using a gradient echo technique with low flip angles (FLASH or fast low-angle shot). No gadolinium contrast-enhanced imaging was acquired as the T1 sequence was primarily used to identify areas of hemorrhage.

\section{Image Analysis}

Three experienced nuclear medicine interpreters, who were masked to the MR imaging and histopathologic results, reached a consensus determination on ${ }^{18} \mathrm{~F}$-DCFBC uptake within the prostate on both a lesion and a sector basis. Visual analysis was used to define focal ${ }^{18} \mathrm{~F}-$ DCFBC uptake as being above background activity in the prostate and blood pool in the periprostatic vascular plexus. For the subsequent analysis, foci of uptake were defined on a 3-point scale as 1, negative; 2, equivocal; or 3, positive. PET maximum standarized uptake values $\left(\mathrm{SUV}_{\max }\right)$ corrected for lean body mass were calculated for the foci of PET uptake in the prostate on the 2D pelvis, 3D pelvis, and WB PET acquisitions using XD Software (Mirada Medical USA). For sites of prostate tumors seen on pathology that were occult on PET, a tumor $\mathrm{SUV}_{\max }$ was obtained using a representative spheric $1-\mathrm{cm}$ diameter volume of interest placed in the approximate tumor location on each PET acquisition, using the prostatectomy specimen as a guide. To evaluate uptake in BPH, the axial T2-weighted MR images were virtually fused to each of the $2 \mathrm{D}$ pelvis, 3D pelvis, and WB PET acquisitions using rigid registration with the femoral heads as fiducial markers on the Mirada Medical workstation. Ellipsoid volumes of interest corresponding to the T2 signal abnormalities deemed to be representative of BPH on the MR images were then drawn, and the $\mathrm{SUV}_{\text {max }}$ for each focus of BPH was determined. Care was taken in drawing the volume of interest of all cases of both tumor and BPH to avoid including any of the high radioactivity in the urinary bladder.

An experienced interpreter of prostate MR images, who was similarly masked to the PET/CT and histopathologic results, evaluated the MR images. From a multiparametric visual analysis including diffusionweighted imaging and high-resolution T1 and T2 imaging, a sector-based determination of the presence of cancer, hemorrhage, $\mathrm{BPH}$, and prostatitis was performed. Lesion-based analysis for the presence of cancer was also performed. Potential sites of cancer were subjectively graded on a 5-point scale (1, highly unlikely to be cancer; 2 , unlikely to be cancer; 3 , equivocal; 4 , likely to be cancer; and 5 , highly likely to be cancer).

\section{Histopathologic Analysis}

All 13 patients who participated in the imaging portion of the study proceeded to have radical prostatectomy with lymph node dissection as is the standard of surgical care at our institution. Briefly, prostate specimens were injected with formalin. Nine were subsequently microwave-fixed, and 4 were fixed in formalin overnight. After fixation, the external surface was differentially inked to indicate the surgical margins per our laboratory standard protocol. The prostatectomy specimens were axially serially sectioned at 4-mm intervals after the proximal and the distal margins and seminal vesicles were removed and entirely submitted for histologic examination. All prostate slices were photographed with a ruler, labeled, and then quartered to produce right anterior, left anterior, right posterior, and left posterior segments; in combination with the base, mid, and apex designations, this method of sectioning the gland provided the basis for the 12-segment model used extensively for lesion localization.

The prostate tissue fragments were embedded in paraffin and stained with hematoxylin and eosin using standard methods. An expert urologic pathologist reviewed the slides and noted the presence of any prostate cancer, Gleason grades, and the size of the tumors. A representative section from the dominant (index) nodule in each prostatectomy specimen was selected for immunohistochemical analysis. The dominant nodule was defined as the nodule with the highest Gleason score tumor, which in most cases was also the largest in size. If a higher Gleason grade was noted in a smaller nodule, that smaller nodule was considered the dominant.

The representative sections from the dominant (index) tumor nodule were then analyzed by immunohistochemical methods for the expression of PSMA, prostate-specific antigen (PSA), ERG, and Ki-67. Appropriate internal and external controls were also processed and assessed in parallel. An $\mathrm{H}$ score was assigned as the sum of the products of the intensity ( 0 for negative, 1 for weakly positive, 2 for moderately positive, and 3 for strongly positive) multiplied by the extent of expression ( $0 \%-$ $100 \%$ ), obtaining a value ranging from 0 to 300 (28). The presence of $\mathrm{Ki}-67$ was reported as a percentage of the tumor cells that demonstrated positive staining. Modified $\mathrm{H}$ scores were calculated for PSMA, taking into account only the strongly and moderately staining portions of the tumor $\left(\mathrm{H}\right.$ score $_{\text {mod-str }}=[$ (percentage of tumor strongly staining $\times 3)+$ (percentage of tumor moderately staining $\times 2$ )]) or only the strongly staining portions of the tumor $\left(\mathrm{H}\right.$ score $_{\text {str }}=$ [percentage of tumor strongly staining $\times 3]$ ). The PSMA $\mathrm{H}$ scores (including $\mathrm{H}$ score $_{\text {mod-str }}$

TABLE 1

Patient Demographic and Clinical Information

\begin{tabular}{|c|c|c|c|c|}
\hline Patient no. & Age $(y)$ & PSA (ng/mL) & Biopsy Gleason score & Prostatectomy Gleason score \\
\hline 1 & 58 & 5.7 & $3+3=6$ & $3+3=6$ \\
\hline 2 & 71 & 17.0 & $4+4=8$ & $4+5=9$ \\
\hline 3 & 65 & 4.5 & $4+3=7$ & $4+3=7$ \\
\hline 4 & 54 & 5.3 & $3+4=7$ & $3+4=7$ \\
\hline 5 & 55 & 13.9 & $4+4=8$ & $4+5=9$ \\
\hline 6 & 57 & 5.6 & $4+3=7$ & $4+5=9$ \\
\hline 7 & 61 & 10.5 & $3+4=7$ & $3+4=7$ \\
\hline 8 & 61 & 9.1 & $4+3=7$ & $4+3=7$ \\
\hline 9 & 71 & 6.8 & $3+4=7$ & $3+4=7$ \\
\hline 10 & 66 & 8.5 & $3+4=7$ & $3+4=7$ \\
\hline 11 & 59 & 5.8 & $4+3=7$ & $5+3=8$ \\
\hline 12 & 62 & 10.5 & $3+4=7$ & $3+4=7$ \\
\hline 13 & 70 & 5.5 & $4+4=8$ & $4+4=8$ \\
\hline
\end{tabular}


TABLE 2

PET and MR Imaging Detection of Prostate Cancer on 12-Segment Prostatectomy Pathology

\begin{tabular}{|c|c|c|c|c|c|}
\hline Modality & Sensitivity & Specificity & $\begin{array}{c}\text { Positive } \\
\text { predictive value }\end{array}$ & $\begin{array}{c}\text { Negative } \\
\text { predictive value }\end{array}$ & Accuracy \\
\hline \multicolumn{6}{|l|}{ MR imaging } \\
\hline All positive & $\begin{array}{l}0.39(0.25-0.54) \\
n=69\end{array}$ & $\begin{array}{l}0.89(0.81-0.94) \\
\quad n=75\end{array}$ & $\begin{array}{l}0.73(0.53-0.87) \\
n=34\end{array}$ & $\begin{array}{l}0.58(0.37-0.77) \\
n=110\end{array}$ & $\begin{array}{c}0.65(0.51-0.76) \\
n=144\end{array}$ \\
\hline Stringent & $\begin{array}{l}0.35(0.20-0.55) \\
\quad n=69\end{array}$ & $\begin{array}{l}0.91(0.82-0.95) \\
\quad n=75\end{array}$ & $\begin{array}{l}0.73(0.50-0.88) \\
\quad n=29\end{array}$ & $\begin{array}{c}0.58(0.36-0.77) \\
n=115\end{array}$ & $\begin{array}{c}0.62(0.48-0.75) \\
n=144\end{array}$ \\
\hline \multicolumn{6}{|c|}{${ }^{18} \mathrm{~F}-\mathrm{DCFBC}$ PET } \\
\hline All positive & $\begin{array}{l}0.17(0.09-0.29) \\
n=77\end{array}$ & $\begin{array}{l}0.96(0.87-0.99) \\
n=79\end{array}$ & $\begin{array}{l}0.81(0.51-0.95) \\
n=16\end{array}$ & $\begin{array}{l}0.53(0.35-0.70) \\
n=140\end{array}$ & $\begin{array}{c}0.57(0.42-0.71) \\
n=156\end{array}$ \\
\hline Stringent & $\begin{array}{l}0.10(0.04-0.25) \\
\quad n=77\end{array}$ & $\begin{array}{c}1.00(1.00-1.00) \\
n=79\end{array}$ & $\begin{array}{l}1.00(1.00-1.00) \\
n=8\end{array}$ & $\begin{array}{c}0.53(0.35-0.70) \\
n=148\end{array}$ & $\begin{array}{c}0.56(0.39-0.71) \\
n=156\end{array}$ \\
\hline
\end{tabular}

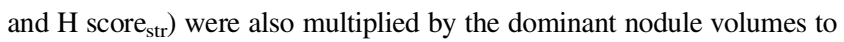
provide additional metrics designated by (H-score $\times$ volume).

\section{Correlation Between Histopathology and Imaging}

Hematoxylin and eosin slides were scanned at 20x using iScan Coreo (Ventana). Photoshop CS6 (Adobe Systems) was used to manually reassemble the prostate quarters into full slices and to register the histology sections to the gross photographs. For each reassembled slice, outlines of tumor, urethra, and prostate gland were then created as layers in Photoshop.

Correlation between histology and the imaging modalities was performed on a per-segment and per-lesion basis. First, the 12-segment prostate model was used, designating each segment containing prostate cancer determined at pathology. Each suggestive finding previously noted on ${ }^{18} \mathrm{~F}$-DCFBC PET and prostate MR imaging interpretation was assigned to 1 or more contiguous segments depending on its location and extent. Additionally, BPH and hemorrhage identified on MR imaging were assigned to segments in which they were present. In that particular set of patients, mild diffuse $\mathrm{T} 2$ signal hypointensity within the prostate that might have indicated prostatitis was not observed. Furthermore, a lesion-based correlation was performed between the dominant tumor on pathology, the corresponding uptake on ${ }^{18} \mathrm{~F}-\mathrm{DCFBC}$ PET, and detection by MR imaging signal abnormality.

\section{Statistical Analysis}

Using the 12-segment model described above, we calculated the sensitivity, specificity, positive predictive value, negative predictive value, and overall accuracy of ${ }^{18} \mathrm{~F}$-DCFBC PET/CT and multiparametric MR imaging with hematoxylin and eosin as the reference standard. Those values were determined with both nonstringent and stringent reading of the imaging modalities, with nonstringent reading defined with equivocal findings ( 2 on PET, 3 on MR imaging) considered to be positive and stringent reading defined with equivocal findings as negative. For that analysis, 1 and 2 on the 5-point scale were considered negative on MR imaging. Estimates of sensitivity, specificity, positive predictive value, negative predictive value, overall accuracy, and their corresponding $95 \%$ confidence limits were derived from intercept-only generalized estimating equation regression models. The same 12-segment model was used to correlate findings of hemorrhage and BPH between PET and MR imaging using a logistic regression model estimated via a generalized estimating equation to arrive at between-group differences. The sensitivity of PET and MR imaging for the detection of the dominant nodule in each patient was also determined on both a nonstringent and a stringent basis.

Several additional correlations were drawn between PET and MR imaging parameters and the pathologic findings. Those included PSMA $H$ score and ( $\mathrm{H}$ score $\times$ volume) metrics versus $2 \mathrm{D}$ pelvis, 3D pelvis, and WB PET $\mathrm{SUV}_{\max }$, PSMA $\mathrm{H}$ score and $(\mathrm{H}$ score $\times$ volume) metrics versus MR imaging ADC values, and PET SUV $\mathrm{Sax}_{\max }$ versus MR imaging ADC values. Nonparametric Pearson correlation coefficients were calculated to describe associations.

Further analysis was performed with uptake in BPH in comparison to the visually positive tumors, both nonstringently and stringently assessed. Generalized estimating equation regression analysis was used to determine between-group differences in the PET uptake among BPH and the visually positive tumors.

\section{RESULTS}

Table 1 includes selected demographic information for the 13 patients recruited. The average age was $62 \mathrm{y}$ (with an SD of $6 \mathrm{y}$ and a range from 54 to $71 \mathrm{y}$ ). The most recent available PSA values at the time of the ${ }^{18} \mathrm{~F}-\mathrm{DCFBC}$ PET/CT scan are also noted, with an average of $8.4 \mathrm{ng} / \mathrm{mL}$ (with an $\mathrm{SD}$ of $3.8 \mathrm{ng} / \mathrm{mL}$ and range from 5.3 to $17.0 \mathrm{ng} / \mathrm{mL}$ ).

A 12-segment prostate analysis for cancer detection with both PET and MR imaging, compared with histology at prostatectomy for tumor, is summarized in Table 2, including nonstringent (rows 1 and 3 ) and stringent (rows 2 and 4) image analyses. Of note,

\section{TABLE 3}

PET and MR Imaging-Dominant Pathology Lesion Detection Sensitivity

\begin{tabular}{cc}
\hline Modality & Sensitivity (95\% confidence interval) \\
\hline MR imaging & \\
All positive & $0.92(0.59-0.99), n=12$ \\
Stringent & $0.75(0.45-0.92), n=12$ \\
${ }^{18}$ F-DCFBC PET & \\
All positive & $0.46(0.22-0.72), n=12$ \\
Stringent & $0.23(0.08-0.52), n=13$ \\
\hline
\end{tabular}


TABLE 4

Dominant Pathology Lesion Size, Gleason Score, and PET Detection

\begin{tabular}{|c|c|c|c|c|}
\hline Dominant nodule size (mL) & Patient no. & $\begin{array}{l}\text { Prostatectomy } \\
\text { Gleason } \\
\text { score }\end{array}$ & $\begin{array}{c}\text { Visually detected } \\
\text { with }{ }^{18} \text { F-DCFBC PET }\end{array}$ & $\mathrm{SUV}_{\max }$ \\
\hline 8.92 & 13 & $4+4=8$ & Equivocal & 3.3 \\
\hline 7.07 & 5 & $4+5=9$ & Yes & 3.5 \\
\hline 5.48 & 12 & $3+4=7$ & No & 1.7 \\
\hline 4.86 & 7 & $3+4=7$ & Yes & 4.6 \\
\hline 2.41 & 2 & $4+5=9$ & Yes & 4.1 \\
\hline 1.23 & 9 & $3+4=7$ & No & 1.4 \\
\hline 1.12 & 6 & $4+5=9$ & Yes & 7.5 \\
\hline 0.73 & 11 & $5+3=8$ & No & 2.3 \\
\hline 0.56 & 10 & $3+4=7$ & No & 1.9 \\
\hline 0.49 & 1 & $3+3=6$ & No & 2.4 \\
\hline 0.44 & 3 & $4+3=7$ & No & 1.1 \\
\hline 0.37 & 4 & $3+4=7$ & No & 1.5 \\
\hline 0.26 & 8 & $4+3=7$ & Equivocal & 2.9 \\
\hline
\end{tabular}

neither ${ }^{18}$ F-DCFBC PET nor MR imaging demonstrated high sensitivity for cancer detection (sensitivities of 0.17 and 0.39 for PET and MR imaging for nonstringent analysis and 0.10 and 0.35 for stringent analysis, respectively), likely reflecting the ability of the interpreting pathologists to identify small to microscopic amounts of tumor in many segments that would be occult on any imaging modality. Although overall accuracy for both modalities using the 12-segment analysis was modest, both ${ }^{18} \mathrm{~F}$-DCFBC PET and MR imaging demonstrated high specificity and positive predictive value for segment-based prostate cancer detection (specificity of 0.96 and 0.89 for PET and MR imaging, respectively, for non-

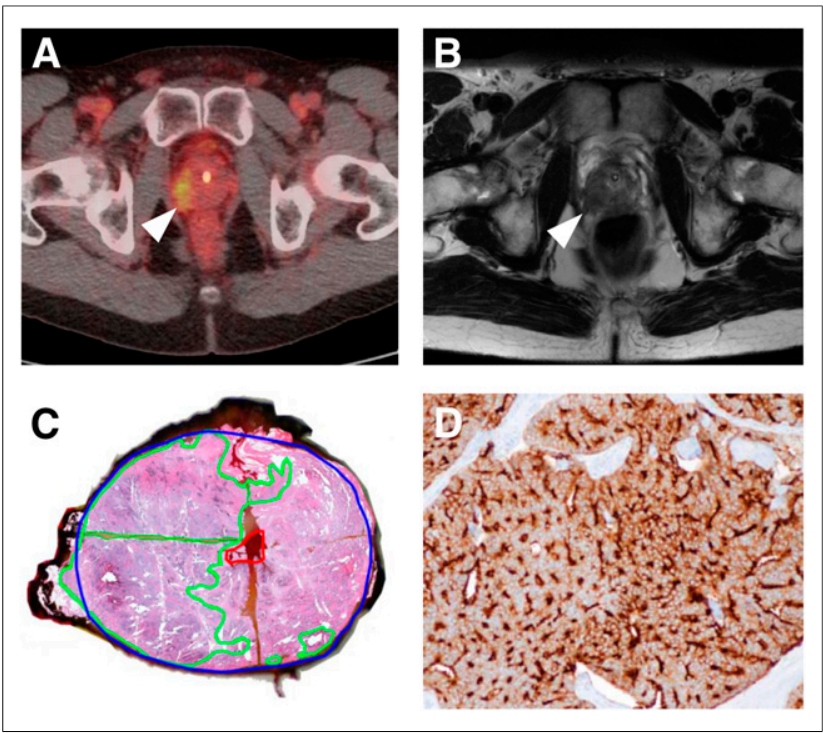

FIGURE 2. Correlation between focal uptake in right lateral prostate apex (arrowhead) on ${ }^{18} \mathrm{~F}$-DCFBC PET (A), abnormal low T2 signal (arrowhead) on MR imaging (B), and tumor on gross surgical pathology, as outlined in green (C). Pathologic specimen from same tumor shows strong immunohistochemical staining for PSMA (brown color) (D). stringent analysis). There was a trend toward ${ }^{18} \mathrm{~F}$-DCFBC PET being marginally more specific than MR imaging in this portion of the analysis ( 0.96 vs. 0.89 for nonstringent analysis and 1.00 vs. 0.91 for stringent analysis, respectively).

In a lesion-based analysis of dominant (index) primary prostate tumor, both modalities had improved sensitivity, compared with the 12-segment analysis, as seen in Table 3, although MR imaging had an increased sensitivity, compared with ${ }^{18} \mathrm{~F}$-DCFBC PET. Specificity was not calculated because of the small sample size of the dominant lesions. The gap in lesion sensitivity between the 2 modalities decreased when the analysis was limited to only Gleason 8 and 9 cancers (MR imaging, 1.00 nonstringent and 0.80 stringent; PET, 0.80 nonstringent and 0.60 stringent). As expected, the sensitivities of both modalities trended lower when equivocal findings were considered negative. Interestingly, the detection of dominant lesions with ${ }^{18} \mathrm{~F}$-DCFBC PET was not solely dependent on tumor size, as would be of concern for any modality in which partial-volume effects can obscure small lesions. Table 4 shows that primary prostate tumor size and Gleason score must both influence lesion detectability by ${ }^{18} \mathrm{~F}$-DCFBC PET as seen by an undetectable Gleason 7 tumor greater than $5 \mathrm{~mL}$ in volume and positive uptake in a smaller 1.1-mL Gleason 9 lesion. Tumors less than $1.1 \mathrm{~mL}$ and Gleason $4+3=7$ were limited in detection by PET, likely in part due to small size and in part to low PSMA expression. On a per-patient basis, as per Table 4, 6 of 13 patients were ${ }^{18} \mathrm{~F}$-DCFBC PET-positive (4 positive on stringent and 6 positive on nonstringent PET reads). All 4 of 4 patients with high-grade (Gleason 8 and 9) and large-volume dominant lesions $(>1.0 \mathrm{~mL})$ were detectable by PET. However, 2 patients with lowvolume $(<1.0 \mathrm{~mL})$ Gleason $4+4=8$ and $4+3=7$ disease and others with lower grade Gleason grade tumors were not detectable by ${ }^{18}$ F-DCFBC PET. Figure 2 shows an example of PET and MR imaging of primary prostate cancer in a patient with a Gleason 9 tumor with corresponding pathology and demonstration of high tumor PSMA expression.

Our original rationale to acquire 2D and 3D pelvic PET images, as well as later WB 3D PET acquisition, was to control for the 
TABLE 5

Average ${ }^{18}$ F-DCFBC Uptake in Positive and Negative Prostate Cancers As Well As Background Blood Pool and Musculature

\begin{tabular}{|c|c|c|c|c|c|c|}
\hline \multirow[b]{2}{*}{ Anatomic site } & \multicolumn{2}{|l|}{$2 \mathrm{D}$ pelvis } & \multicolumn{2}{|l|}{ 3D pelvis } & \multicolumn{2}{|l|}{ WB } \\
\hline & Average $\mathrm{SUV}_{\max }$ & SD & Average $\mathrm{SUV}_{\max }$ & SD & Average $\mathrm{SUV}_{\max }$ & SD \\
\hline $\begin{array}{l}\text { Prostate cancer with positive } \\
\text { uptake }(n=6)\end{array}$ & 4.7 & 1.2 & 3.3 & 0.6 & 4.3 & 1.7 \\
\hline $\begin{array}{l}\text { Prostate cancer with negative } \\
\text { uptake }(n=7)\end{array}$ & 1.8 & 0.6 & 1.9 & 0.8 & 1.7 & 0.5 \\
\hline $\begin{array}{l}\text { Blood pool (right common femoral } \\
\text { vasculature) }\end{array}$ & 3.8 & 0.8 & 2.5 & 0.4 & 2.1 & 0.2 \\
\hline Muscle (right gluteus musculature) & 2.0 & 0.5 & 1.0 & 0.2 & 0.9 & 0.1 \\
\hline
\end{tabular}

possibility of increased scatter from the urinary bladder radioactivity into the adjacent prostate gland on 2D compared with 3D images. However, that phenomenon was not observed to any measurable extent visually or quantitatively, as seen in Table 5. Visually, the later WB images demonstrated the highest tumor-tobackground normal prostate and blood-pool uptake with improved lesion conspicuity, most likely as a result of their later acquisition time, which allowed some background blood-pool radioactivity to clear with respect to ${ }^{18} \mathrm{~F}-\mathrm{DCFBC}$-mediated radioactivity within the tumor (Table 5). ${ }^{18} \mathrm{~F}-\mathrm{DCFBC}$ PET tumor-to-blood-pool ratio (tumor $\mathrm{SUV}_{\max }$ to blood-pool $\mathrm{SUV}_{\text {mean }}$ ) using the right common femoral vein for blood pool demonstrated this trend of increasing tumor-to-background ratio on later WB PET acquisitions (Supplemental Fig. 1; supplemental materials are available at http://jnm. snmjournals.org). Given those considerations, we chose to perform all subsequent analyses using the standardized uptake values derived from the WB PET acquisitions obtained at the longest duration after ${ }^{18} \mathrm{~F}$-DCFBC injection, unless otherwise specified (time interval of $\sim 2.5 \mathrm{~h}$ after injection). Notably, there were no incidental findings on the WB PET images in any patients to suggest metastatic disease.

In reviewing the data from the 3 different PET acquisitions (2D pelvis, 3D pelvis, and $\mathrm{WB}$ ), it was observed that there was a statistically significant positive correlation $(P<0.05)$ between the Gleason scores of the tumors and the obtained maximum standardized uptake values for all 3 acquisitions (Fig. 3). We observed nearly no relationship between Gleason score and ADC values in our study (Supplemental Fig. 2). When correlating $\mathrm{SUV}_{\max }$ to PSMA expression (PSMA $\mathrm{H}$ score, PSMA $\mathrm{H}$ score $_{\text {mod-str, }}$ and PSMA $\mathrm{H}$ score $_{\text {str }}$ ), positive relationships were noted for all 3 PSMA immunohistochemical scores with a trend toward but no statistical significance (Supplemental Fig. 3, $\rho$ values between 0.31 and 0.51;
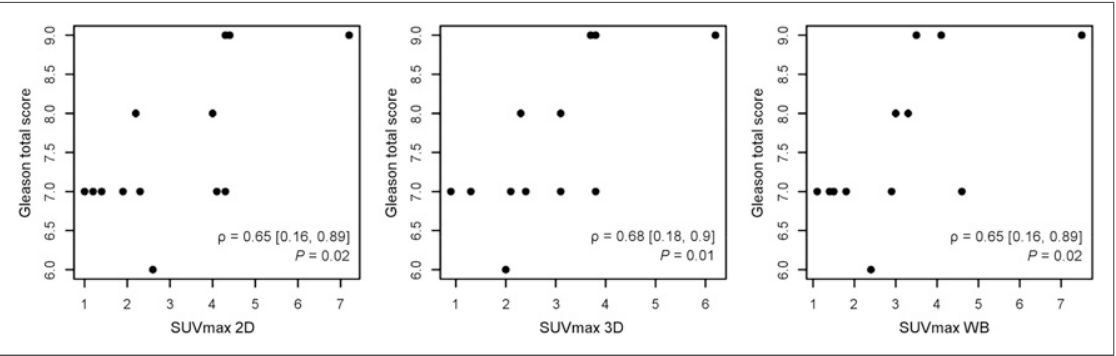

FIGURE 3. Scatterplot of ${ }^{18} \mathrm{~F}-\mathrm{DCFBC}$ PET SUV $\mathrm{max}_{\text {ax }}$ and prostatectomy Gleason score for pelvic $2 \mathrm{D}$, pelvic $3 \mathrm{D}$, and WB PET acquisitions showing strong positive correlation.
$P$ value of $0.1,0.07$, and 0.3 , respectively). In regards to non-PSMA immunohistochemical findings, we observed a positive correlation between PSA H score and $\mathrm{SUV}_{\max }$, a negative correlation between ERG $\mathrm{H}$ score and $\mathrm{SUV}_{\max }(\rho-0.31)$, and a negative correlation between Ki-67 staining and $\operatorname{SUV}_{\max }(\rho-0.28)$ (Supplemental Fig. 3 ); none of these associations reached statistical significance. More details on these correlations, as well as correlation of MR imaging $\mathrm{ADC}$ to immunohistochemical parameters, are presented in the supplemental data section.

Figure 4 shows the relative photopenia we observed in $\mathrm{BPH}$, compared with the rest of the prostate gland. The central gland is noted to have 2 large BPH nodules. BPH does not express PSMA and does not demonstrate focal uptake with ${ }^{18} \mathrm{~F}$-DCFBC. Across all of the imaged BPH lesions and PET-positive tumors, there is a statistically significant difference in uptake between BPH and PET-positive prostate cancers (Fig. 5, $P=0.004$ and 0.016 , respectively).

\section{DISCUSSION}

Major considerations in the management of prostate cancer are accurate initial diagnosis and distinguishing aggressive from indolent disease for selection of appropriate therapy. Patient care initially requires accurate tumor evaluation to select the optimal therapy from a growing array of alternatives that include active surveillance, androgen ablation, radical prostatectomy (radical retropubic or laparoscopic/robotic), radiation therapy (brachytherapy, external-beam radiation therapy, or combinations of these choices), and possibly focal ablative therapies (cryoablation, radiofrequency ablation, brachytherapy, laser ablation, and focused ultrasound) $(3,29,30)$. Patients are risk-stratified based on serum PSA level, tumor grade, and clinical stage, with predictive models having been developed to determine pathologic stage and time to recurrence based on retrospective patient data (31). However, those outcome models, while effective, do not adequately identify all patients at risk of developing biochemical recurrence and provide no anatomic localization of tumor spread (32).

The combined anatomic and functional imaging provided by PET suggests that a PET radiotracer for the proper target may dramatically improve imaging of prostate cancer. Studies with ${ }^{18}$ F-FDG, the most 


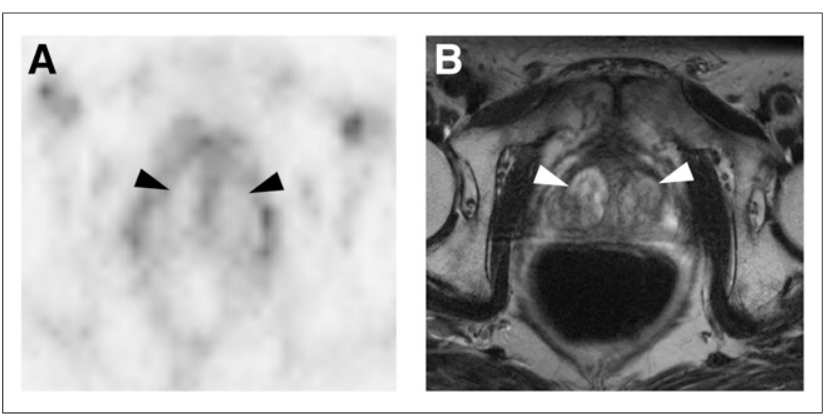

FIGURE 4. ${ }^{18} \mathrm{~F}-\mathrm{DCFBC} \mathrm{PET}(\mathrm{A})$ and T2-weighted MR (B) images demonstrating ${ }^{18} \mathrm{~F}$-DCFBC photopenia for representative example of $\mathrm{BPH}$ nodules (arrowheads) within central prostate.

commonly used clinical PET radiotracer, have demonstrated low uptake in prostate cancer except for advanced metastatic disease $(33,34)$. However, several new radiotracers for prostate cancer are in various stages of development as noted in the introduction. In particular, choline, acetate, and ${ }^{18} \mathrm{~F}-\mathrm{FACBC}$ PET imaging have been hampered by decreased specificity in differentiating malignant from benign hyperplastic prostatic lesions $(11,12,14)$, although the PET radiotracer synthetic bombesin receptor antagonist for gastrin-releasing peptide was able to differentiate between malignant and benign hyperplastic prostate lesions (18).

PSMA is a promising, well-characterized biomarker specific for prostate cancer, which has also been associated with prostate tumor aggressiveness. Histologic studies have associated high PSMA expression with metastatic spread (35-37) and androgen independence (38), and expression levels have been found to be predictive of prostate cancer progression $(19,21)$.

Our prospective study evaluated the utility of ${ }^{18} \mathrm{~F}-\mathrm{DCFBC}$, a smallmolecule PSMA inhibitor, for the detection of primary prostate cancer. We were able to detect clinically significant high-grade (Gleason 8 and 9$)$ and larger-sized ( $\geq 1.1 \mathrm{~mL}$ ) primary prostate tumors reliably, with no evidence of uptake in BPH. ${ }^{18} \mathrm{~F}-\mathrm{DCFBC}$ PET was, however, limited for detection of smaller-sized $(<1.1 \mathrm{~mL})$ and lower-grade (Gleason 7 or 6) tumors. Although low-grade tumors were found to have variable and generally low-level uptake, there was nevertheless a positive correlation between ${ }^{18} \mathrm{~F}$-DCBFC uptake, as measured by $\mathrm{SUV}_{\max }$ and Gleason score of the prostate cancers included in this trial. We have observed a trend toward a positive correlation between ${ }^{18}$ F-DCFBC PET tumor uptake, compared with PSMA tumor expression by immunohistochemistry, although a study with a larger sample

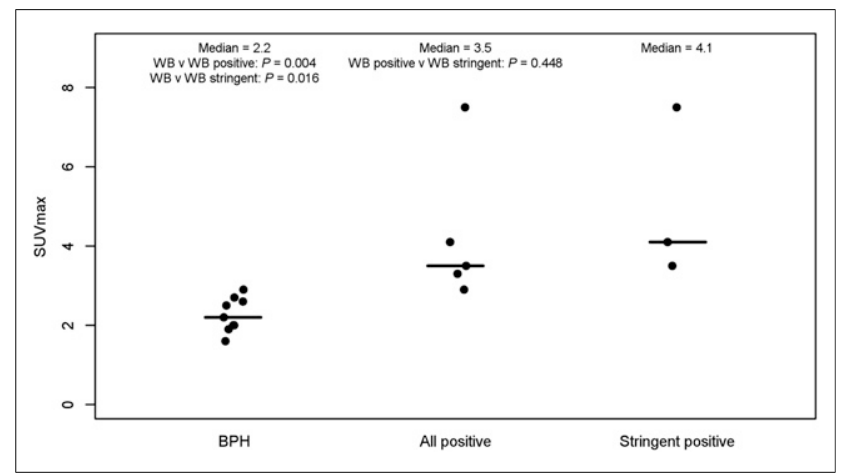

FIGURE 5. Plot showing ranges of SUV $\max$ in BPH, all PET-positive prostate cancers (nonstringent analysis), and tumors with PET positivity only in stringent analysis. size is needed to confirm these preliminary results. MR imaging demonstrated greater sensitivity for the detection of prostate cancer but can oftentimes reveal multiple lesions whereas ${ }^{18} \mathrm{~F}$-DCFBC PET can potentially allow for more specific localization of the highestgrade and most clinically significant lesion.

This study had several limitations. First, the overall detection rate of prostate cancer by ${ }^{18} \mathrm{~F}$-DCFBC PET/CT in our patient cohort was lower than multiparametric MR imaging, although the detection rate improved with higher-grade tumors (Gleason 8 and 9). We were limited by the accrual pattern of patients who entered our study having predominantly low-grade tumors (Gleason 6 and 7), which are known to have relatively low PSMA expression levels $(19,21)$. Attempting to include more patients with tumors of higher Gleason grade in the initial recruitment process would likely have improved our rate of detection. Another important consideration is that many tumors in the prostate are small (significantly $<1 \mathrm{~cm}$ in diameter), rendering them susceptible to partial-volume effects. However, when correcting for lesion size we showed that the detection of dominant lesions with ${ }^{18}$ F-DCFBC PET was not solely dependent on tumor size but also involved tumor PSMA expression. It is likely that small tumors may have been volume-averaged with surrounding normal prostate tissue, preventing their detection on PET imaging in patients with low-grade tumors and corresponding low levels of PSMA expression.

Another consideration arises from the intrinsic properties of ${ }^{18} \mathrm{~F}$ DCFBC. ${ }^{18} \mathrm{~F}-\mathrm{DCFBC}$ is a first-generation ${ }^{18} \mathrm{~F}$-labeled agent that demonstrated high and specific uptake in PSMA-expressing experimental models and in patients with metastatic disease $(22,23) .{ }^{18} \mathrm{~F}$ DCFBC tends to persist in the blood pool, likely secondary to plasma protein binding, which limits optimal clearance from soft tissue and diminishing tumor-to-background ratio and detection of lower avidity or smaller lesions. ${ }^{68} \mathrm{Ga}$-PSMA (39) and a new secondgeneration ${ }^{18} \mathrm{~F}$-PSMA-targeted agent $\left({ }^{18} \mathrm{~F}\right.$-DCFPyL) (40) demonstrate high tumor uptake with lower-background, which promises improved signal for better detection of lower-grade or smaller-sized primary prostate tumors, compared with ${ }^{18} \mathrm{~F}$-DCFBC. The low ${ }^{18} \mathrm{~F}-$ DCFBC PET uptake in BPH shown in this study demonstrates that these emerging PSMA-based PET imaging agents can also potentially greatly improve the specificity for differentiating benign hyperplastic from malignant prostate lesions, which has also been an important reported feature of a new gastrin-releasing peptide receptor radiopharmaceutical (18) but a limitation of acetate, choline, and ${ }^{18}$ F-FACBC PET imaging for this application $(11,12,14)$.

\section{CONCLUSION}

${ }^{18}$ F-DCFBC PET can detect clinically significant, high-grade prostate cancer and shows promise for differentiating malignant prostate cancer from nonmalignant prostate lesions such as BPH. With further validation, PSMA-targeted PET imaging, in conjunction with MR imaging, may allow for directed biopsy of the most clinically significant lesions and function as a noninvasive imaging biomarker for differentiating indolent versus aggressive disease, thus improving risk-adaptive management.

\section{DISCLOSURE}

The costs of publication of this article were defrayed in part by the payment of page charges. Therefore, and solely to indicate this fact, this article is hereby marked "advertisement" in accordance with 18 USC section 1734. Funding was provided by the Prostate Cancer Foundation (PCF) and Movember (the PCF Creativity Award), the 
Brady Patana Research Fund, T32 EB006351, and CA134675. No other potential conflict of interest relevant to this article was reported.

\section{ACKNOWLEDGMENTS}

We thank Akimosa Jeffrey-Kwanisai and Yavette Morton for their dedication to, and clinical coordination of, this trial. The ClinicalTrials.gov Identifier number is NCT01496157. Dr. Kenneth L. Gage's current affiliation is the H. Lee Moffitt Cancer Center, Tampa, Florida.

\section{REFERENCES}

1. Siegel R, Ma J, Zou Z, Jemal A. Cancer statistics, 2014. CA Cancer J Clin. 2014;64:9-29.

2. Cuzick J, Thorat MA, Andriole G, et al. Prevention and early detection of prostate cancer. Lancet Oncol. 2014;15:e484-e492.

3. Chang AJ, Autio KA, Roach M 3rd, Scher HI. High-risk prostate cancer-classification and therapy. Nat Rev Clin Oncol. 2014;11:308-323.

4. Johnson LM, Turkbey B, Figg WD, Choyke PL. Multiparametric MRI in prostate cancer management. Nat Rev Clin Oncol. 2014;11:346-353.

5. Dianat SS, Carter HB, Macura KJ. Performance of multiparametric magnetic resonance imaging in the evaluation and management of clinically low-risk prostate cancer. Urol Oncol. 2014;32:39 e1-10.

6. Bonekamp D, Jacobs MA, El-Khouli R, Stoianovici D, Macura KJ. Advancements in MR imaging of the prostate: from diagnosis to interventions. Radiographics. 2011;31:677-703.

7. Kiess AP, Cho SY, Pomper MG. Translational molecular imaging of prostate cancer. Curr Radiol Rep. 2013;1:216-226.

8. Jadvar H. Molecular imaging of prostate cancer with PET. J Nucl Med. 2013;54:1685-1688.

9. Farsad M, Schiavina R, Castellucci P, et al. Detection and localization of prostate cancer: correlation of ${ }^{11} \mathrm{C}$-choline PET/CT with histopathologic step-section analysis. J Nucl Med. 2005;46:1642-1649.

10. Kwee SA, Thibault GP, Stack RS, Coel MN, Furusato B, Sesterhenn IA. Use of step-section histopathology to evaluate ${ }^{18} \mathrm{~F}$-fluorocholine PET sextant localization of prostate cancer. Mol Imaging. 2008;7:12-20.

11. Mena E, Turkbey B, Mani H, et al. ${ }^{11} \mathrm{C}$-acetate PET/CT in localized prostate cancer: a study with MRI and histopathologic correlation. J Nucl Med. 2012;53:538-545.

12. Souvatzoglou M, Weirich G, Schwarzenboeck $S$, et al. The sensitivity of $\left[{ }^{11} \mathrm{C}\right]$ choline PET/CT to localize prostate cancer depends on the tumor configuration. Clin Cancer Res. 2011;17:3751-3759.

13. Schuster DM, Taleghani PA, Nieh PT, et al. Characterization of primary prostate carcinoma by anti-1-amino-2-[ $\left.{ }^{18} \mathrm{~F}\right]$-fluorocyclobutane-1-carboxylic acid (anti-3$\left[{ }^{18} \mathrm{~F}\right]$ FACBC) uptake. Am J Nucl Med Mol Imaging. 2013;3:85-96.

14. Turkbey B, Mena E, Shih J, et al. Localized prostate cancer detection with ${ }^{18} \mathrm{~F}$ FACBC PET/CT: comparison with MR imaging and histopathologic analysis. Radiology. 2014;270:849-856.

15. Foss CA, Mease RC, Cho SY, Kim HJ, Pomper MG. GCPII imaging and cancer. Curr Med Chem. 2012;19:1346-1359.

16. Huang C, McConathy J. Fluorine-18 labeled amino acids for oncologic imaging with positron emission tomography. Curr Top Med Chem. 2013;13:871-891.

17. Wieser G, Mansi R, Grosu AL, et al. Positron emission tomography (PET) imaging of prostate cancer with a gastrin releasing peptide receptor antagonist: from mice to men. Theranostics. 2014;4:412-419.

18. Kähkönen E, Jambor I, Kemppainen J, et al. In vivo imaging of prostate cancer using $\left[{ }^{68} \mathrm{Ga}\right]-$ labeled bombesin analog BAY86-7548. Clin Cancer Res. 2013; 19:5434-5443.

19. Ross JS, Sheehan CE, Fisher HA, et al. Correlation of primary tumor prostatespecific membrane antigen expression with disease recurrence in prostate cancer. Clin Cancer Res. 2003;9:6357-6362.
20. Marchal C, Redondo M, Padilla M, et al. Expression of prostate specific membrane antigen (PSMA) in prostatic adenocarcinoma and prostatic intraepithelial neoplasia. Histol Histopathol. 2004;19:715-718.

21. Perner S, Hofer MD, Kim R, et al. Prostate-specific membrane antigen expression as a predictor of prostate cancer progression. Hum Pathol. 2007;38:696-701.

22. Foss CA, Mease RC, Fan H, et al. Radiolabeled small-molecule ligands for prostate-specific membrane antigen: in vivo imaging in experimental models of prostate cancer. Clin Cancer Res. 2005;11:4022-4028.

23. Cho SY, Gage KL, Mease RC, et al. Biodistribution, tumor detection, and radiation dosimetry of ${ }^{18} \mathrm{~F}-\mathrm{DCFBC}$, a low-molecular-weight inhibitor of prostatespecific membrane antigen, in patients with metastatic prostate cancer. $J \mathrm{Nucl}$ Med. 2012;53:1883-1891.

24. Kozikowski AP, Nan F, Conti P, et al. Design of remarkably simple, yet potent urea-based inhibitors of glutamate carboxypeptidase II (NAALADase). J Med Chem. 2001;44:298-301

25. Mease RC, Dusich CL, Foss CA, et al. $N$-[N-[(S)-1,3-dicarboxypropyl $]$ carbamoyl]-4-[ $\left[{ }^{18} \mathrm{~F}\right]$ fluorobenzyl-L-cysteine, $\left[{ }^{18} \mathrm{~F}\right] \mathrm{DCFBC}$ : a new imaging probe for prostate cancer. Clin Cancer Res. 2008;14:3036-3043.

26. Holt DPRH, Mathews WB, Horti A, Mease R, Dannals RF. A semi-automated microwave chemistry system for complete radiosynthesis, purification, and formulation of F-18 radiotracers. J Label Compd Radiopharm. 2011;54.

27. Yao V, Berkman CE, Choi JK, O'Keefe DS, Bacich DJ. Expression of prostatespecific membrane antigen (PSMA), increases cell folate uptake and proliferation and suggests a novel role for PSMA in the uptake of the non-polyglutamated folate, folic acid. Prostate. 2010;70:305-316.

28. Chaux A, Albadine R, Toubaji A, et al. Immunohistochemistry for ERG expression as a surrogate for TMPRSS2-ERG fusion detection in prostatic adenocarcinomas. Am J Surg Pathol. 2011;35:1014-1020.

29. Sartor O, Eisenberger M, Kattan MW, Tombal B, Lecouvet F. Unmet needs in the prediction and detection of metastases in prostate cancer. Oncologist. 2013;18: 549-557.

30. Walsh PC, DeWeese TL, Eisenberger MA. Clinical practice: localized prostate cancer. N Engl J Med. 2007;357:2696-2705.

31. Han M, Partin AW, Zahurak M, Piantadosi S, Epstein JI, Walsh PC. Biochemical (prostate specific antigen) recurrence probability following radical prostatectomy for clinically localized prostate cancer. J Urol. 2003;169:517-523.

32. Cowen ME, Halasyamani LK, Kattan MW. Predicting life expectancy in men with clinically localized prostate cancer. J Urol. 2006;175:99-103.

33. Turlakow A, Larson SM, Coakley F, et al. Local detection of prostate cancer by positron emission tomography with 2-fluorodeoxyglucose: comparison of filtered back projection and iterative reconstruction with segmented attenuation correction. Q J Nucl Med. 2001;45:235-244.

34. Hofer C, Laubenbacher C, Block T, Breul J, Hartung R, Schwaiger M. Fluorine18-fluorodeoxyglucose positron emission tomography is useless for the detection of local recurrence after radical prostatectomy. Eur Urol. 1999;36:31-35.

35. Bostwick DG, Pacelli A, Blute M, Roche P, Murphy GP. Prostate specific membrane antigen expression in prostatic intraepithelial neoplasia and adenocarcinoma: a study of 184 cases. Cancer. 1998;82:2256-2261.

36. Chang SS, Reuter VE, Heston WD, Gaudin PB. Comparison of anti-prostatespecific membrane antigen antibodies and other immunomarkers in metastatic prostate carcinoma. Urology. 2001;57:1179-1183.

37. Sweat SD, Pacelli A, Murphy GP, Bostwick DG. Prostate-specific membrane antigen expression is greatest in prostate adenocarcinoma and lymph node metastases. Urology. 1998;52:637-640.

38. Wright GL Jr, Grob BM, Haley C, et al. Upregulation of prostate-specific membrane antigen after androgen-deprivation therapy. Urology. 1996;48:326-334.

39. Afshar-Oromieh A, Malcher A, Eder M, et al. PET imaging with a $\left[{ }^{68} \mathrm{Ga}\right]$ galliumlabelled PSMA ligand for the diagnosis of prostate cancer: biodistribution in humans and first evaluation of tumour lesions. Eur J Nucl Med Mol Imaging. 2013;40:486495.

40. Szabo Z, Mena E, Rowe SP, et al. Initial evaluation of [F]DCFPyL for prostatespecific membrane antigen (PSMA)-targeted PET imaging of prostate cancer. Mol Imaging Biol. April 21, 2015 [Epub ahead of print]. 